and contraction-excitation coupling. Singling out a few papers for mention, Tonomura and co-workers summarize and interpret their data on the kinetics of the interaction of ATP analogues with myosin systems. Kielley reviews his evidence for the existence of three polypeptide chains in myosin and for the arrangement of the meromyosin moieties within the molecule.

The role of the bound nucleotide of actin remains a challenging issue, and Oosawa and his colleagues conclude that, while not indispensable, it does exert a large effect on actin polymerization. They also contribute new evidence that conformational changes in the actin polymer occur during contraction.

A salutary warning to biochemists, who perforce are often obliged to use mixtures of red and white muscle, is given by Gergely and his co-workers. They find very large differences in the properties of fragmented sarcoplasmic reticulum, of the myofibrils and even of the purified myosin obtained from red and white fibres. They make the interesting suggestion that red muscle myosin may be related to cardiac myosin. Finally, Ebashi discusses how the action potential arriving at the central tubule of a triad could elicit the release of calcium at the lateral vesicles.

My main criticism of this book is that the contributions are specialist in nature and are not general reviews. Thus the book cannot be recommended to students and others unfamiliar with the field, whereas there certainly is a need for a modern text-book on the subject.

\section{G. W. OFFER}

\section{VETERINARY CODEX}

British Veterinary Codex 1965

Pp. xxxv +843. (London: The Pharmaceutical Press, 1965.) 1058.

THe long-awaited new edition of this book follows closely the layout and content of its predecessor. As an authoritative reference book for those concerned with the manufacture, supply and use of drugs in veterinary practice, it will no doubt continue to be of great value. It is unfortunate, however, that an interval of twelve years should elapse between editions even when an interim supplement is published.

The Codex consists of three main parts; monographs on drugs and chemicals, on antisera and vaccines, and a formulary. This is followed by seventeen appendixes and a therapeutic index.

Part I has been extensively revised by the addition of 56 new monographs, while 106 have now been deleted. Several drugs in current use are not included. I was struck in particular by the absence of griseofulvin, while acepromazine, bendrofluazide, framycetin, heparin, phencyclidine, tripelennamine and tylosine are also missing. Conversely, is it still necessary to include camphor, chenopodium and rhubarb? The monographs are generally useful and informative although the description of the administration of halothane (p. 180) did not impress. The potential toxic hazards of stilboestrol implantation in lambs are not mentioned, although similar effects in cattle and poultry are noted.

Part II (biological products) has been revised and contains several additions. Four new appendixes have been added, including a useful section on pharmaceutical adjuvants. The appendixes on feed supplements and gasliquid chromatography are, however, too short to be of any great value. Brief descriptions of complex subjects are rarely satisfactory and adequate enlargement could scarcely be justified in a veterinary codex. The therapeutic index is also of limited value, although it could be useful for reference or possibly in an emergency.

This is an essential publication which would benefit from more frequent revision, so that changes in the field of veterinary therapeutics can be more rapidly assimilated.

J. SANFORD

\section{FROM WOAD TO WEED}

\section{Weeds of the World}

Biology and Control. By Lawrence J. King. (Plant Science Monographs.) Pp. xxxii $+526+23$ plates. (London: Leonard Hill; New York: Interscience Publishers, Inc., 1966.) $105 s$ net.

THIs book is another volume in the Plant Science Monograph series, which is designed primarily to bring together the scattered literature of important specialities in the plant sciences, with the ultimate and indirect object of helping to feed and clothe the increasing world population and to improve productivity and hence living standards. There can be no doubt of the suitability of weeds as a subject in this series, nor of the need of a review of research in this subject. The present book, announced at least five years before publication, aroused high expectations, so that it is a pity to have to register a measure of disappointment.

The first eleven chapters are devoted to weed biology in the widest possible sense of both words. One of the objects of these monographs is to cover all aspects of the subject concerned, and Dr. King has certainly attempted to do this, even to the extent of listing some of the more extraordinary uses to which man has put weeds, from using a Lithospermum for contraception to an Angelica root as a talisman for gamblers. The chapters are a series of reviews, each with its relevant bibliography, and the author has made much use of original publications, especially for their graphical and pictorial material. In the first chapter there is a lengthy consideration of the words used for weeds in several languagos, from the possible origin of "weed" from "woad" (Isatis tinctoria) in English, to the interpretation of Chinese and Japanese ideographs. Various definitions of weeds follow and these too are considered in detail.

Having started in such detail and diversity, it is perhaps not suprising to find a certain imbalance in the volume. Matters that might have furthered the aims of this series, such as the significance and increasing importance of rhizomatous weeds in certain systems and areas of agriculture, are mentioned only in scattered references, whereas the importance of weed seeds is well considered, presumably in view of the author's association with the Boyce Thompson Institute.

The four chapters on weed control could well have been omitted: they add nothing to existing works and are noticeably dated. For example, in a book published in 1966, where (apart from one list in an appendix) are the bipyridyls? In addition, the naming of herbicides is inconsistent and the primary authors are not always quoted. This is the case with the grouping of triazines on structural affinity.

Despite these criticisms this is a valuable book, which will undoubtedly be read to great profit by all those interested in weeds, for it refers frequently to earlier biological or more purely botanical publications that are not always appreciated or even discovered by those involved in more practical aspects. R. J. Chancellor

\section{PLANT LATIN}

\section{Botanical Latin}

History, Grammar, Syntax, Terminology and Vocabulary. By William T. Stearn. Pp. xiv +566 . (Edinburgh: Thomas Nelson and Sons, Ltd., 1966.) $105 s$.

Srstematic botany is probably the only discipline which uses Latin as a regular, indeed compulsory, means of 